potentially protective enzyme increases rapidly. Finally, CTL surface cathepsin B was shown to be enzymatically active and to cleave perforin efficiently.

This work indicates that cytotoxic lymphocytes release granule-derived cathepsin B on degranulation, which associates with the cell surface and protects these cells against perforin attack and self-destruction. So, a long-standing question about the granule-exocytosis model seems to have been answered.

Jenny Buckland, Associate Editor, Nature Reviews Immunology

\section{6) References and links}

ORIGINAL RESEARCH PAPER Balaji, K. et al.

Surface cathepsin B protects cytotoxic

lymphocytes from self-destruction after

degranulation. J. Exp. Med. 196, 493-503

(2002)

FURTHER READING Barry, M. \& Bleackley, R. C. Cytotoxic T lymphocytes: all roads lead to death. Nature Rev. Immunol. 2, 401-409 (2002)

Pierre Henkart's lab:

http://www3.cancer.gov/intra/EIB/henkart.htm

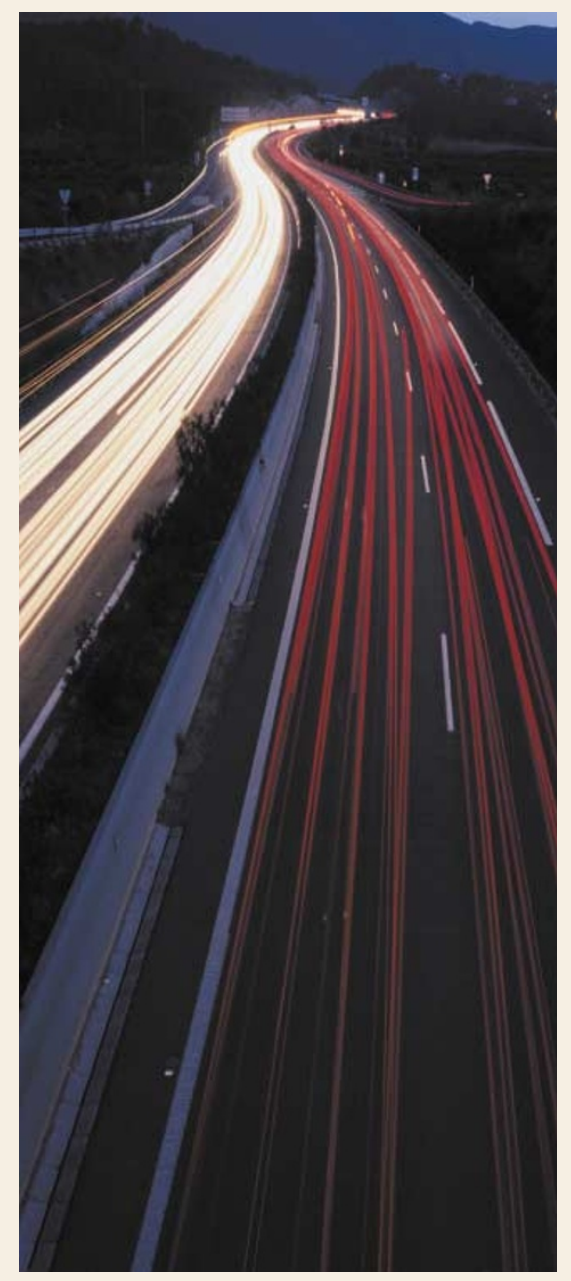

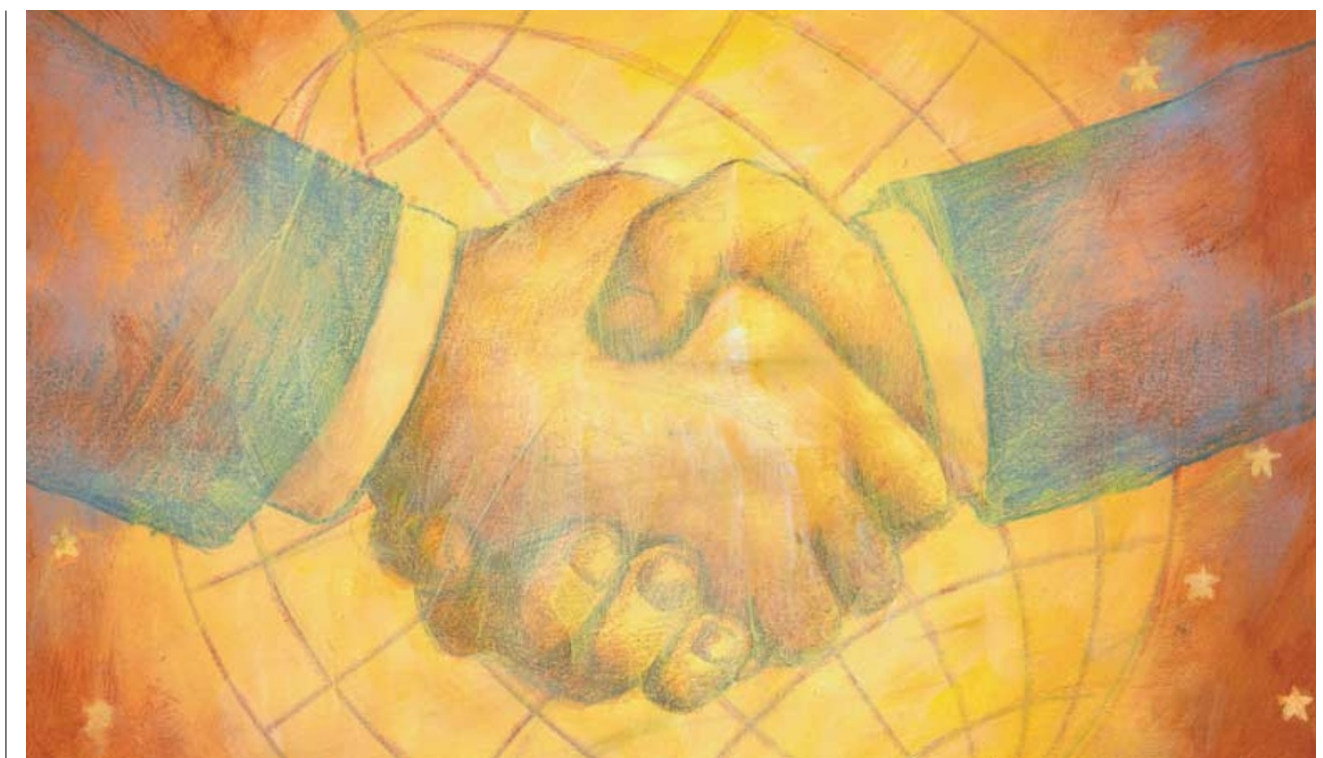

CELL ADHESION

\title{
Promoting partnerships
}

Some areas of cell biology, despite a wealth of data, remain confusing. So far, the role of Rac in adhesion has been one such area, but James Nelson's group now propose a function for Rac in the kinetics and strengthening of E-cadherin-mediated cell-cell adhesion.

To study membrane activity dynamically at cell-cell contacts, Nelson's group used time-lapse phase-contrast microscopy, and saw that exploratory lamellipodia were responsible for the initial contacts that were made between MDCK cells. Once opportunistic collisions between cells had occurred, a local burst of lamellipodia was seen at the intercellular contact sites.

The small Rho GTPase Rac regulates the formation of lamellipodia, so, to be sure of Rac's involvement, the authors used green fluorescent protein (GFP)-tagged Rac1. GFP-Rac1 localized to the tips of extending lamellipodia in migrating cells, but once cell-cell contact had been made, it accumulated at the newly contacting membranes. Over time, though, as the contact area expanded, lamellipodia formation decreased, as did the intensity of the GFP-Racl signal at the original cell-cell contact site. Concomitant with the increase in contact area, the authors noticed a relative decrease in the number of lamellipodia that were originally present around the cell periphery.

As is common when studying small GTPases, dominant-negative and constitutively active constructs were used. Cell-cell contacts in cells that expressed dominant-negative (T17N) Rac grew very slowly. And not only did fewer lamellipodia form, but the ones that did form weren't restricted to cell-cell contacts alone, so the 'relative protrusive index' decreased. By contrast, constitutively active Rac induced increased lamellipodia formation. As neither construct prevented E-cadherin accumulation at cell-cell contacts, the changes in intercellular adhesion seemed to result from Rac1-dependent actin-based membrane protrusions rather than Ecadherin distribution. But Nelson and colleagues raised the possibility that, by increasing lamellipodia formation, the resultant increase in surface area between the contacting membranes probably increases the likelihood of E-cadherin-mediated cell-cell adhesion.

Finally, Nelson's group analysed Rac's role in the strength of adhesion using a 'hanging drop' cell-cell adhesion assay. Here, cells are forced together, and the sizes of cell aggregates and their resistance to trituration (breaking up of cell clumps) are measured. Consistent with impaired cell-cell contact formation, T17NRac cell aggregates were weaker, and developed resistance to trituration more slowly than their wild-type counterparts. But, despite the altered kinetics and strength of cell-cell adhesion, intercellular contacts did eventually form, indicating that Rac's primary function, through lamellipodia, might be to increase the rate of contact formation and strengthening.

(2) References and links

Katrin Bussell

ORIGINAL RESEARCH PAPER Ehrlich, J. S., Hansen, M. D. H. \& Nelson, W. J. Spatio-temporal regulation of Rac1 localization and lamellipodia dynamics during epithelial cell-cell adhesion. Dev. Cell $\mathbf{3}$, 259-270 (2002)

FURTHER READING Fukata, M. \& Kaibuchi, K. Rho-family GTPases in cadherin-mediated cell-cell adhesion. Nature Rev. Mol. Cell Biol. 2. 887-897 (2001) 